\title{
Postnatal Development of the Retina in Rats Exposed to Hyperoxia: A Fractal Analysis
}

\author{
Anne Claudia Ştefănuţ, ${ }^{1}$ Ştefan Ţălu, ${ }^{2}$ Viorel Miclăuş, ${ }^{3}$ \\ Adriana Mureşan, ${ }^{4}$ Remus Moldovan, ${ }^{4}$ and Bianca Szabo ${ }^{5}$ \\ ${ }^{1}$ Clinic Emergency Hospital Cluj-Napoca, 3-5 Clinicilor Street, 400006 Cluj-Napoca, Romania \\ ${ }^{2}$ Technical University of Cluj-Napoca, Faculty of Mechanical Engineering, Department of AET, \\ Discipline of Descriptive Geometry and Engineering Graphics, 103-105 B-dul Muncii Street, 400641 Cluj-Napoca, Romania \\ ${ }^{3}$ University of Agricultural Sciences and Veterinary Medicine, Faculty of Veterinary Medicine, Department of Cell Biology, \\ Histology and Embriology, 3-5 Mănăştur Street, 400372 Cluj-Napoca, Romania \\ 4 "Iuliu Haţieganu" University of Medicine and Pharmacy Cluj-Napoca, Faculty of Medicine, Department of Physiology, \\ 1 Clinicilor Street, 400006 Cluj-Napoca, Romania \\ 5 "Iuliu Haţieganu" University of Medicine and Pharmacy Cluj-Napoca, Faculty of Medicine, Department of Anatomy, \\ Embryology, Ophthalmology, 3-5 Clinicilor Street, 400006 Cluj-Napoca, Romania
}

Correspondence should be addressed to Ştefan Ţălu; stefan_ta@yahoo.com

Received 27 February 2013; Accepted 25 March 2013

Academic Editors: R. Tadeusiewicz and B. Zheng

Copyright (C) 2013 Anne Claudia Ştefănuț et al. This is an open access article distributed under the Creative Commons Attribution License, which permits unrestricted use, distribution, and reproduction in any medium, provided the original work is properly cited.

\begin{abstract}
Purpose. The aim of this study was to investigate and quantify changes in the newborn rats retinal layers during the hyperoxia $\left(80 \% \mathrm{O}_{2}\right)$ exposure using fractal analysis. Materials and Methods. This study was conducted on two groups of 20 newborn rats: a control (normal) group (10 rats) and an experimental group (10 rats). The control group was composed of 10 newborn rats, which were placed at 12 hours after birth, in a pediatric incubator, together with their mother, in conditions of normoxia for 21 days. The experimental group consisted of 10 newborn rats, which were placed at 12 hours after birth, in a pediatric incubator with their mother, in conditions of normoxia for 7 days, then 7 days of hyperoxia $\left(80 \% \mathrm{O}_{2}\right)$ for 22.5 hours/day, and then 7 days in conditions of normoxia. Slaughtering of the rats was performed on day 21 and the eye globes were harvested in order to perform histopathological examinations. The fractal analyses of the retinal digital images were performed using the fractal analysis software Image J, and the fractal dimensions were calculated using the standard box-counting method. Results. Microscopic examination revealed a normal development of the retina in the control group. In the experimental group, all the animals exposed to hyperoxia revealed both structural and vascular abnormalities on entire retina. Conclusions. The results showed that the fractal analysis is a valuable tool to quantify histoarchitectural changes in the newborn rats retinal layers during the hyperoxia $\left(80 \% \mathrm{O}_{2}\right)$.
\end{abstract}

\section{Introduction}

In the last few decades important efforts have been directed to understand various aspects of the newborn rats retinal layers, for clinical diagnostics and treatment [1-4].

The rat's retina is immature at birth, being comparable with that from human fetuses of 26 weeks, and allows tracking its development postnatally [1]. Exposure to oxigen of rat pups retina, during the first days after birth, develops an oxygen-induced retinopathy (OIR), similar to human retinopathy of prematurity (ROP) [2-4]. Retinopathy of prematurity is a leading cause of blindness in children [5-9].

Newborn rat model represents a solution to study normal and abnormal development of primary visual system of human subjects [4]. The cycle hyperoxia-normoxia mimics ischemia-reperfusion and leads to retinal neovascularization $[2-4,10-12]$. This experimental model could be useful in the study of angiogenesis occurring in other potentially blinding retinal diseases (e.g., diabetes), cancer, and degenerative diseases and in the study of the effect of some substances with 
antioxidant and/or antiangiogenic potential, which stimulate the normal development of the retina [12].

Computerized image visualization and advances in analysis methods of the retinal layers using the fractal geometry is a part of the early detection and diagnosis of retinal diseases and can be useful for describing the pathological architecture of retina [13-16].

The fractal analysis is applied to the fractal objects that cannot be measured properly using regular Euclidean geometry [13-16]. The irregular shapes of cells, tumors, and vasculature defy description by traditional Euclidean geometry, which is based on smooth shapes (such as the line, plane, cylinder, cone, and sphere). In practice a fractal object is statistically similar on magnification over a finite range of length scales. The self-similarity of biological objects is maintained in a "scaling window," which normally ranges in two to three orders of magnitude [14].

Fractal dimensions of image objects can be measured using computational approaches. Fractal dimension quantifies the degree of complexity into a single value, being a measure of how the fractal object fills up space. The fractal dimension measures a qualitative feature of fractal geometric objects that is used to distinguish different types of fractals.

The fractal analysis depends on the experimental and methodological parameters involved as diversity of subjects, image acquisition, type of image, image processing, fractal analysis methods, including the algorithm and specific calculation used, and so forth [14, 15, 17-22].

\section{Materials and Methods}

2.1. General Methodology. The experimental study was conducted at the "Iuliu Haţieganu" University of Medicine and Pharmacy Cluj-Napoca, in the Research Center of the Department of Physiology. The protocol was approved by the Ethics Committee of "Iuliu Haţieganu" University of Medicine and Pharmacy Cluj-Napoca [12].

In this study we used white rats, Wistar breed, females, and newborn rat 12 hours old, with birth weight of 10 grams. The animals came from Biobase of "Iuliu Haţieganu" University of Medicine and Pharmacy Cluj-Napoca and they were kept in vivarium conditions appropriate at the Department of Physiology. These were placed in an Isolette incubator, in which temperature was controlled at 23-24 degrees, lightdark cycle of 12 hours (using artificial white light 200 lux).

Females received a standard normocaloric diet, and water ad libitum and the newborn rats were fed by lactating mother. Toilet incubator was made for periods of $3 \times 0.5$ hours/day.

The experimental study was conducted on 20 animals divided into 2 equal lots for 21 days. Control group (normoxia) was performed in 10 newborn rats put on 12 hours of birth, along with their mother in an incubator, type Isolette, where they provided normoxic conditions for 21 days $(21 \%$ $\mathrm{O}_{2}$ ) (Days 0-21).

Consignment of animals exposed to hyperoxia was performed continuously in 10 rats put on 12 hours of birth, along with their mother in an incubator under conditions identical to those of control group (normoxia) for 7 days (Days 0-7).
In the next 7 days (Days 8-14) the animals were exposed to hyperoxia, daily to an oxygen concentration of $80 \% \mathrm{O}_{2}$ for 22.5 hours/day. After exposure to hyperoxia, the incubator was again assured normoxic conditions for 7 days, from Day 15 until Day 21.

To achieve hyperoxic condition we used a mobile oxygen concentrator adapted to the incubator, with a flow rate $=$ $1.5 \mathrm{~L} / \mathrm{min}$. Oxygen concentration was monitored twice/day using an electronic gas analyzer.

2.2. Histopathological Examination. Processing of tissue samples and retinal histopathology were performed at the University of Agricultural Sciences and Veterinary Medicine, Faculty of Veterinary Medicine, Laboratory of the Department of Histology, Cluj-Napoca. The histopathological examinations of the enucleated eyes were performed on 21 day. Serial sections of $5 \mu \mathrm{m}$ thickness were cut and stained with modified Masson-Goldner trichrome. Examination of stained sections was done by Olympus BX41 microscope.

2.3. Theory of Fractal Analysis. In mathematical calculation, box counting or box dimension is the most common method used to estimate the fractal dimension [23].

The lower and upper box-counting dimensions of a subset $F \subset R^{n}$ are, respectively, defined by [23]

$\underline{\operatorname{dim}}_{B}(F)=\varliminf_{\delta \rightarrow 0} \frac{\log N_{\delta}(F)}{-\log \delta}, \quad \overline{\operatorname{dim}}_{B}(F)=\varlimsup_{\delta \rightarrow 0} \frac{\log N_{\delta}(F)}{-\log \delta}$.

If these are equal then the common value is referred to as the box-counting dimension of $F$ and is denoted by [23]

$$
\operatorname{dim}_{B}(F)=\lim _{\delta \rightarrow 0} \frac{\log N_{\delta}(F)}{-\log \delta}
$$

(if this limit exists), where $N_{\delta}(F)$ is any of the following: (i) the smallest number of closed balls of radius $\delta>0$ that cover $F$; (ii) the smallest number of cubes of side $\delta$ that cover $F$; (iii) the number of $\delta$-mesh cubes that intersect $F$; (iv) the smallest number of sets of diameter at most $\delta$ that cover $F$; (v) the largest number of disjoint balls of radius $\delta$ with centers in $F$.

Twenty digital images were analyzed from each group. After adjustments of every digital image, a binarization on a binary image corresponding to the analyzed structure was obtained. The binary template, which is also referred to as structural element is associated to the binary image using logical operations [24]. The analyzed structure ( 8 bit) was extracted using the morphologic operations from the original digitalized images. This procedure was applied for all analyzed structures.

In our study the box-counting algorithm was performed using the Image J software (Wayne Rasband, National Institutes of Health, in Bethesda, Maryland, USA) [25] together with the FracLac plug-in (A. Karperien, Charles Sturt University, Australia) [26].

The algorithm was applied with the following options: (a) grid positions $(n=10)$; (b) calculating of grid calibers using default box sizes. The range of box sizes used for the fractal 


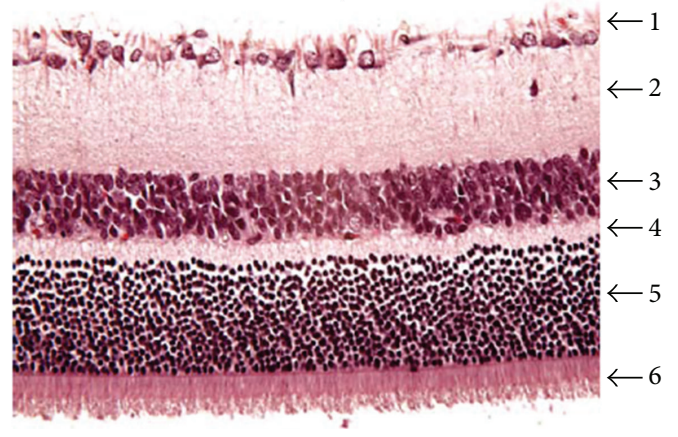

Figure 1: Control group (Goldner's Trichrome ob. $40 \mathrm{X}$ ): (1) cells ganglionar layer CGL; (2) internal plexiform layer IPL; (3) nuclear intern layer INL; (4) outer plexiform layer OPL; (5) outer nuclear layer ONL; (6) photoreceptors layer PL.

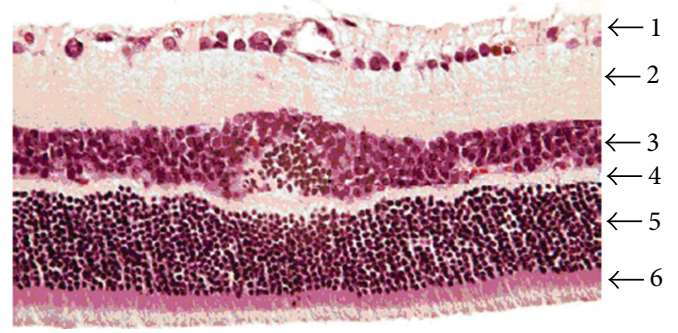

Figure 2: $\mathrm{O}_{2}$ exposed group (Goldner's Trichrome ob. 40X): (1) cells ganglionar layer CGL; (2) internal plexiform layer IPL; (3) internal nuclear layer INL; (4) outer plexiform layer OPL; (5) outer nuclear layer ONL; (6) photoreceptors layer PL.

dimension calculation was 2 pixels (the minimum size box) and $45 \%$ from region of interest (the maximum size box).

The fractal dimension was calculated as the slope of the regression line for the log-log plot of the scanning box size and the count from a box-counting scan. The "count" usually refers to the number of grid boxes that contained pixels in a box-counting scan. The slope of the linear region of the plot is $(-D)$, where $D$ is the box-counting dimension that corresponds to the fractal dimension.

The statistical processing of the results obtained with Image J software was done using GraphPad InStat software program, version 3.20 (GraphPad, San Diego, CA, USA) [27]. Normal distribution of variables was previously assessed by means of the Kolmogorov-Smirnov test. Differences with a $P$ value of 0.05 or less were considered statistically significant. The average results were expressed as mean value and standard deviation.

\section{Results}

In the control group, all histological sections made showed a normal aspect of the retinal vessels and retinal layers (Figure 1).

Retinal sections made of the animals exposed to hyperoxia showed structural abnormalities in both retinal periphery and center (Figure 2). Thus, we have observed zonal thickening of the retina and thinning/thickening zonal retinal
TABLE 1: Results of the fractal dimensions (mean \pm standard deviation) and correlation coefficients $\left(R^{2}\right)$ of analyzed digital retinal layers for normal $(\mathrm{N})$ and pathological $(\mathrm{P})$ groups.

\begin{tabular}{lccc}
\hline Retinal layer & Type & $\begin{array}{c}\text { Fractal } \\
\text { dimensions }(D) \\
\text { (mean } \pm \text { standard } \\
\text { deviation) }\end{array}$ & $\begin{array}{c}\text { Correlation } \\
\text { coefficients } \\
\left(R^{2}\right)\end{array}$ \\
\hline Cells ganglionar layer CGL & $\mathrm{N}$ & $1.3250 \pm 0.0123$ & 0.9990 \\
& $\mathrm{P}$ & $1.2230 \pm 0.0127$ & 0.9980 \\
Internal plexiform layer IPL & $\mathrm{N}$ & $1.7196 \pm 0.0121$ & 0.9981 \\
& $\mathrm{P}$ & $1.6686 \pm 0.0125$ & 0.9980 \\
Internal nuclear layer INL & $\mathrm{N}$ & $1.7268 \pm 0.0124$ & 0.9986 \\
& $\mathrm{P}$ & $1.6048 \pm 0.0123$ & 0.9990 \\
Outer nuclear layer ONL & $\mathrm{N}$ & $1.7655 \pm 0.0122$ & 0.9981 \\
& $\mathrm{P}$ & $1.7437 \pm 0.0125$ & 0.9981 \\
Photoreceptors layer PL & $\mathrm{N}$ & $1.6912 \pm 0.0126$ & 0.9984 \\
& $\mathrm{P}$ & $1.5381 \pm 0.0127$ & 0.9953 \\
\hline
\end{tabular}

Note: $(D)$ : fractal dimension; $\left(R^{2}\right)$ : correlation coefficient.

layers (PL, ONL, INL), loss of parallel disposition ordered to these retinal layers, and folds and cavities in the PL and ONL.

Cytoarchitectural anomalies highlighted in this study emphasized the destructive effect on immature, developing retina after exposure to hyperoxia.

A summary of the obtained results is presented in Table 1.

For all analyzed cases, the coefficients of correlation $\left(R^{2}\right)$ were more than 0.9950 representing good linear correlation. An $\left(R^{2}\right)$ of 1.0 indicates that the regression line perfectly fits the data.

(1) Cells ganglionar layer CGL (see Figure 3).

(2) Internal plexiform layer IPL (see Figure 4).

(3) Internal nuclear layer INL (see Figure 5).

(4) Outer nuclear layer ONL (see Figure 6).

(5) Photoreceptors layer PL (see Figure 7).

\section{Discussion}

Some remarks can be obtained concerning the results from Table 1:

(i) the average of fractal dimensions $(D)$ of the pathological retinal layers is slightly lower than the normal cases;

(ii) the fractal analysis was in agreement with the histological observations.

To obtain a more robust diagnostic tool, we think that fractal dimension together with other morphometric parameters could be an improved, powerful aid in understanding and discriminating pathological retinal layers. Based on this reason, the fractal dimension should be included in a global index considering different parameters. 


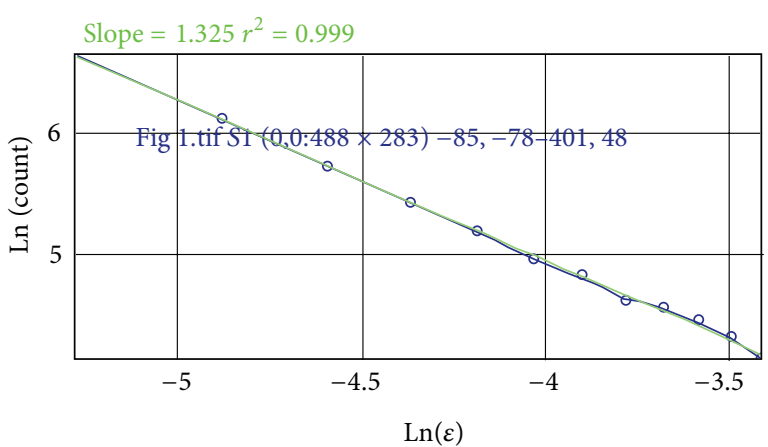

(a)

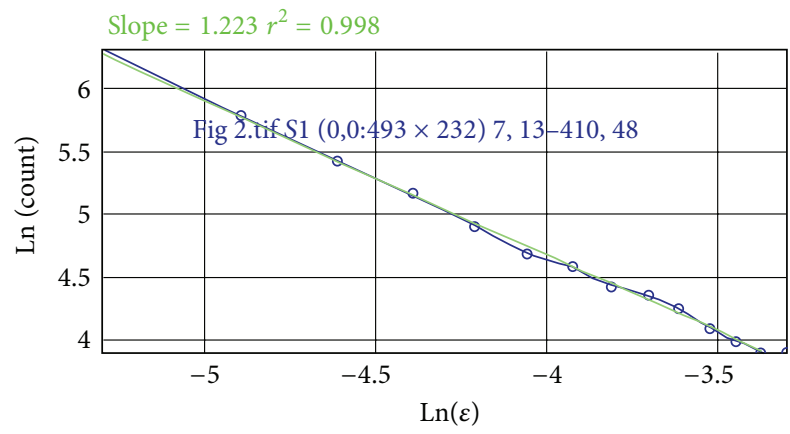

(b)

FIGURE 3: The $\operatorname{Ln}$ (count) versus $\operatorname{Ln}(\varepsilon)$ cells ganglionar layer CGL: (a) normal and (b) pathological status.

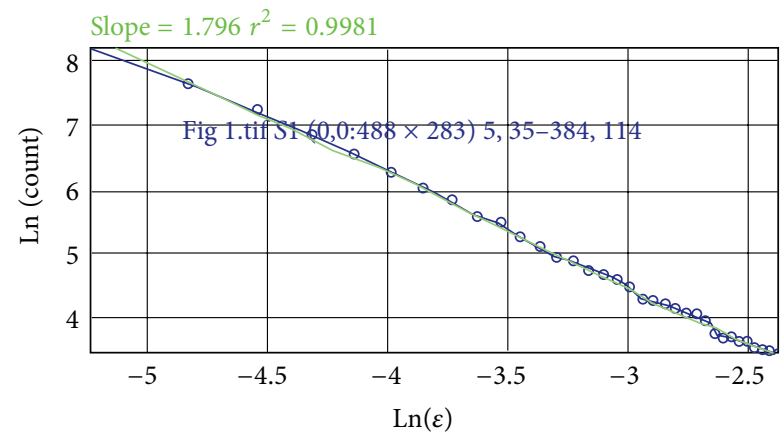

(a)

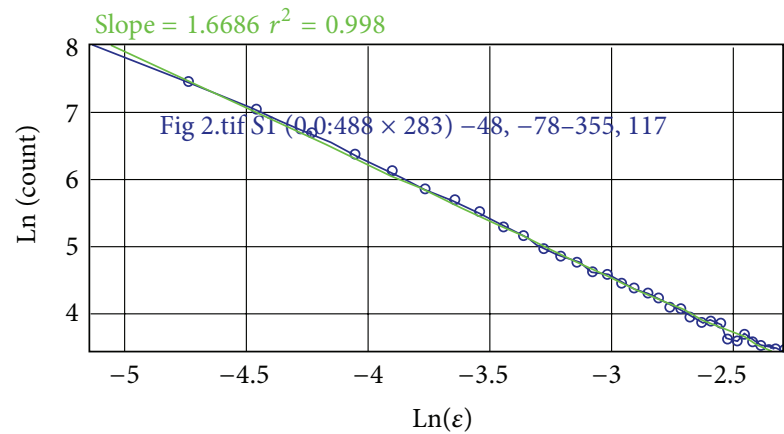

(b)

FIGURE 4: The $\operatorname{Ln}$ (count) versus $\operatorname{Ln}(\varepsilon)$ internal plexiform layer IPL: (a) normal and (b) pathological status.

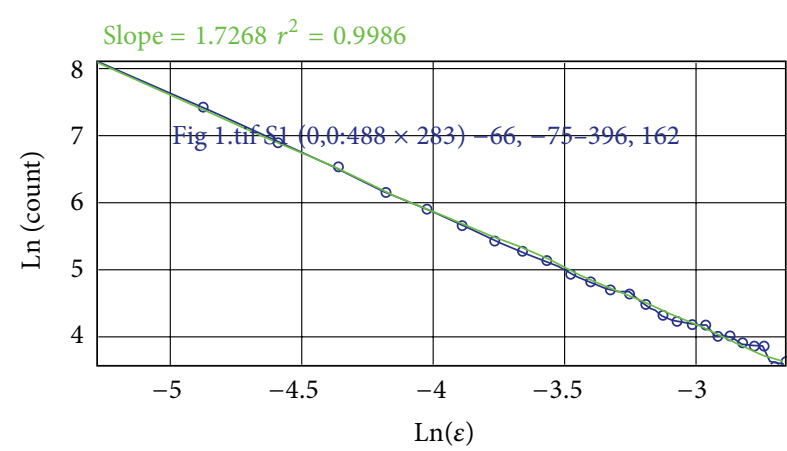

(a)

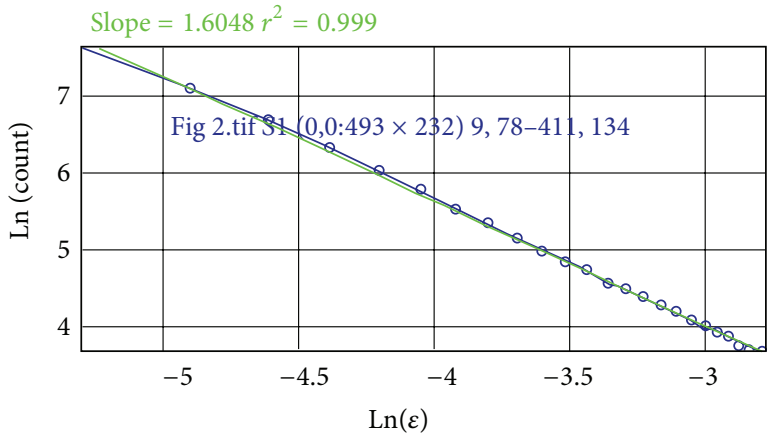

(b)

FIGURE 5: The $\operatorname{Ln}$ (count) versus $\operatorname{Ln}(\varepsilon)$ internal nuclear layer INL: (a) normal and (b) pathological status.

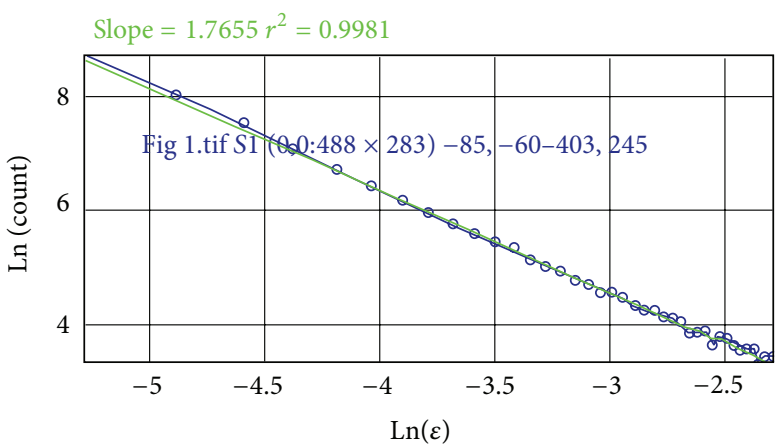

(a)

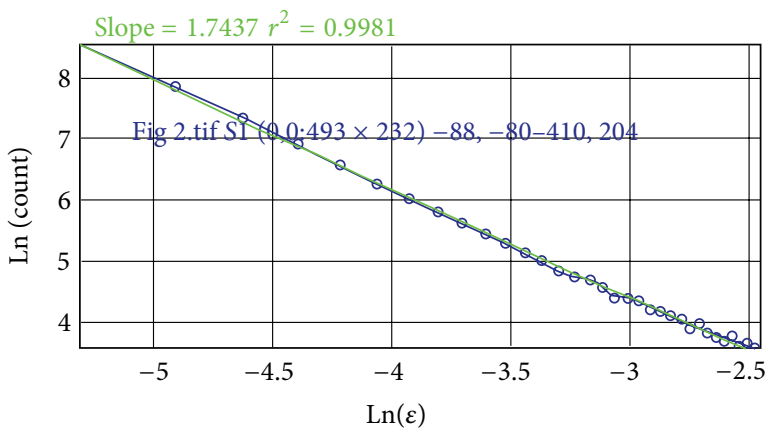

(b)

FIgURE 6: The $\operatorname{Ln}$ (count) versus $\operatorname{Ln}(\varepsilon)$ outer nuclear layer ONL: (a) normal and (b) pathological status. 


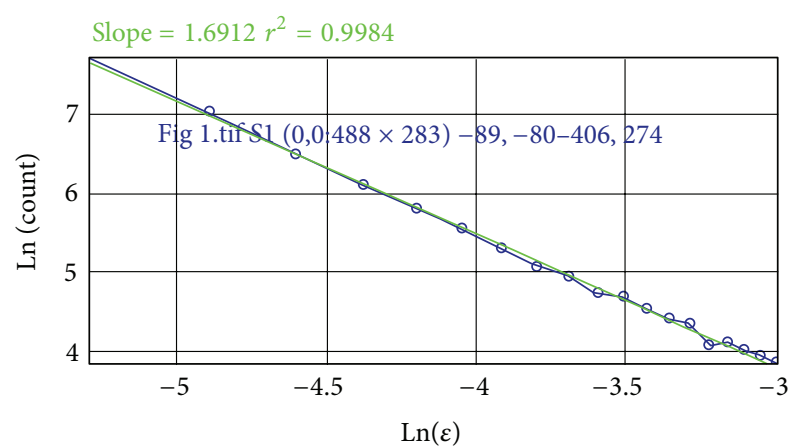

(a)

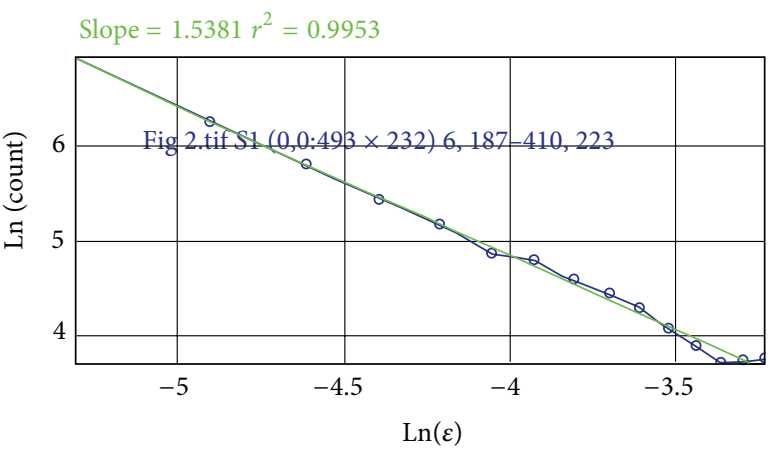

(b)

FIgURE 7: The $\operatorname{Ln}$ (count) versus $\operatorname{Ln}(\varepsilon)$ photoreceptors layer PL: (a) normal and (b) pathological status.

\section{Conclusions}

The fractal analysis may describe the complexity of biological structures that cannot be sufficiently described using the classical Euclidian geometric terms [14].

The fractal analysis is valuable addition to quantify histoarchitectural changes in the newborn rats retinal layers during the hyperoxia $\left(80 \% \mathrm{O}_{2}\right)$.

Emerging data show that variation in the fractal dimensions may serve as an indicator or predictive factor in normal versus pathological conditions, serving as an objective means to guide the researchers.

The fractal analysis may be a tool for examining the mechanistic origins of pathological forms and may someday have a significant impact on our understanding of challenges in treatment delivery and diagnosis of retinal diseases.

\section{Disclosure}

None of the authors has a financial or proprietary interest in any material or method mentioned. The authors alone are responsible for the content and writing of the paper.

\section{References}

[1] J. E. Cairns, "Normal development of the hyaloid and retinal vessels in the rat," The British Journal of Ophthalmology, vol. 43, pp. 385-393, 1959.
[2] A. Dorfman, O. Dembinska, S. Chemtob, and P. Lachapelle, "Early manifestations of postnatal hyperoxia on the retinal structure and function of the neonatal rat," Investigative Ophthalmology and Visual Science, vol. 49, no. 1, pp. 458-466, 2008.

[3] B. Ricci, "Oxygen-induced retinopathy in the rat model," Documenta Ophthalmologica, vol. 74, no. 3, pp. 171-177, 1990.

[4] A. C. Stefănuț, V. Miclăuş, A. Mureșan et al., "Retinal neovascularization în newborn rats submitted to variations of concentrations of oxygen-histopathological aspects," The Bulletin of University of Agricultural Sciences and Veterinary Medicine ClujNapoca, vol. 67, no. 1, pp. 305-315, 2010.

[5] S. D. Ţălu, Ophtalmologie-Cours, Medical Publishing House "Iuliu Haţieganu", Cluj-Napoca, Romania, 2005.

[6] S. D. Ţălu, G. Zaharia, and G. Vasile, "Retinopathy of prematurity: screening and therapeutic indications," Oftalmologia, vol. 49, no. 1, pp. 12-16, 2005.

[7] G. Zaharia, S. D. Țălu, A. C. Stefănuț, C. Vlăduţiu, M. Popa, and L. K. Szbadi, "Assessment of retinopathy of prematurity," Oftalmologia, vol. 51, no. 4, pp. 83-88, 2007.

[8] S. D. Țălu, G. Zaharia, C. Vlăduţiu, A. C. Stefănuţ, and M. Popa, "Laser photocoagulation in retinopathy of prematuritypreliminary results," Oftalmologia, vol. 52, no. 1, pp. 90-94, 2008.

[9] S. D. Ţălu, D. Cormos, G. Zaharia, A. C. Stefănuţ, M. Popa, and D. I. Lucaci, "Prognostic factors for laser treatment in retinopathy of prematurity," Oftalmologia, vol. 55, no. 1, pp. 84-89, 2011.

[10] A. C. Stefănuţ, V. Miclăuş, A. Mureșan et al., "Retinal cytoarchitectural anomalies of retinal development în oxygeninduced retinopathy in Wistar rats pups," Annals of the Romanian Society for Cell Biology Cluj-Napoca, vol. 15, no. 1, pp. 166174, 2010.

[11] V. Miclăuş, A. C. Stefănuţ, A. Mureşan, C. Ober, and V. Rus, "Comparative testing of some experimental models of oxygen induced retinopathy în young rats," Histological Study, Iaşi, vol. 53, no. 12, pp. 107-115, 2010.

[12] A. C. Stefănuţ, Oxidative stress implications in the retinopathy of prematurity etiopathogenesis [Ph.D. thesis], "Iuliu Haţieganu" University of Medicine and Pharmacy, Cluj-Napoca, Romania, 2011.

[13] I. S. Reljin and B. D. Reljin, "Fractal geometry and multifractals in analyzing and processing medical data and images," Archive of Oncology, vol. 10, no. 4, pp. 283-293, 2002.

[14] "Fractals in biology and medicine," in Mathematics and Bioscience in Interaction, G. A. Losa, D. Merlini, T. F. Nonnenmacher, and E. Weibel, Eds., vol. 4, Birkhäuser, Basel, Switzerland, 2005

[15] Ş. Țălu, "Mathematical methods used in monofractal and multifractal analysis for the processing of biological and medical data and images," Animal Biology \& Animal Husbandry, vol. 4, no. 1, pp. 1-4, 2012.

[16] Ş. Ţălu, "Texture analysis methods for the characterisation of biological and medical images," Extreme Life, Biospeology of Astrobiology, vol. 4, no. 1, pp. 8-12, 2012.

[17] R. Lopes and N. Betrouni, "Fractal and multifractal analysis: a review," Medical Image Analysis, vol. 13, no. 4, pp. 634-649, 2009.

[18] Ş. Ţălu and S. Giovanzana, "Fractal and multifractal analysis of human retinal vascular network: a review," Human \& Veterinary Medicine, vol. 3, no. 3, pp. 205-212, 2011.

[19] Ş. Ţălu, "Fractal analysis of normal retinal vascular network," Oftalmologia, vol. 55, no. 4, pp. 11-16, 2011. 
[20] Ş. Ţălu and S. Giovanzana, "Image analysis of the normal human retinal vasculature using fractal geometry," Human \& Veterinary Medicine, vol. 4, no. 1, pp. 14-18, 2012.

[21] Ş. Ţălu, "The influence of the retinal blood vessels segmentation algorithm on the monofractal dimension," Oftalmologia, vol. 56, no. 3, pp. 73-83, 2012.

[22] Ş. Ţălu, "Multifractal characterization of human retinal blood vessels," Oftalmologia, vol. 56, no. 2, pp. 63-71, 2012.

[23] K. Falconer, Fractal Geometry: Mathematical Foundations and Applications, John Wiley \& Sons, Chichester, UK, 2nd edition, 2003.

[24] "Fundamentals of biomedical image processing," in Biomedical Image Processing, T. M. Deserno, Ed., pp. 18-19, Springer, Berlin, Germany, 2011.

[25] “Image J software, version ImageJ 1.47d," Wayne Rasband, National Institutes of Health, Bethesda, Md, USA, http://imagej.nih .gov/ij/.

[26] A. Karperien, "FracLac V 2.0f for Image J software," Charles Sturt University, Victoria, Australia, http://rsbweb.nih.gov/ij/ plugins/fraclac/FLHelp/Introduction.htm.

[27] "GraphPad InStat software, version 3.20," GraphPad, San Diego, Calif, USA, http://www.graphpad.com/instat/instat.htm. 

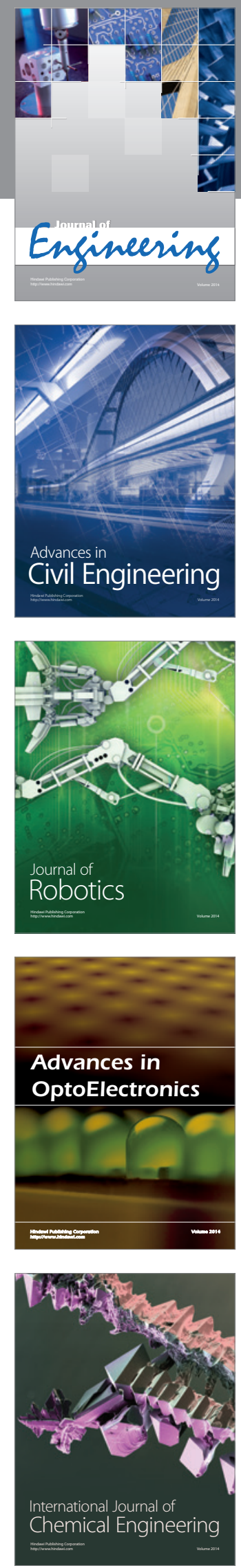

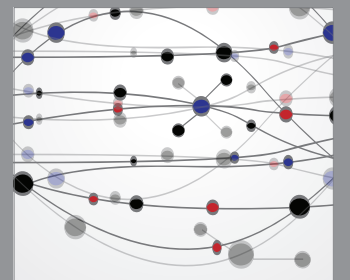

The Scientific World Journal
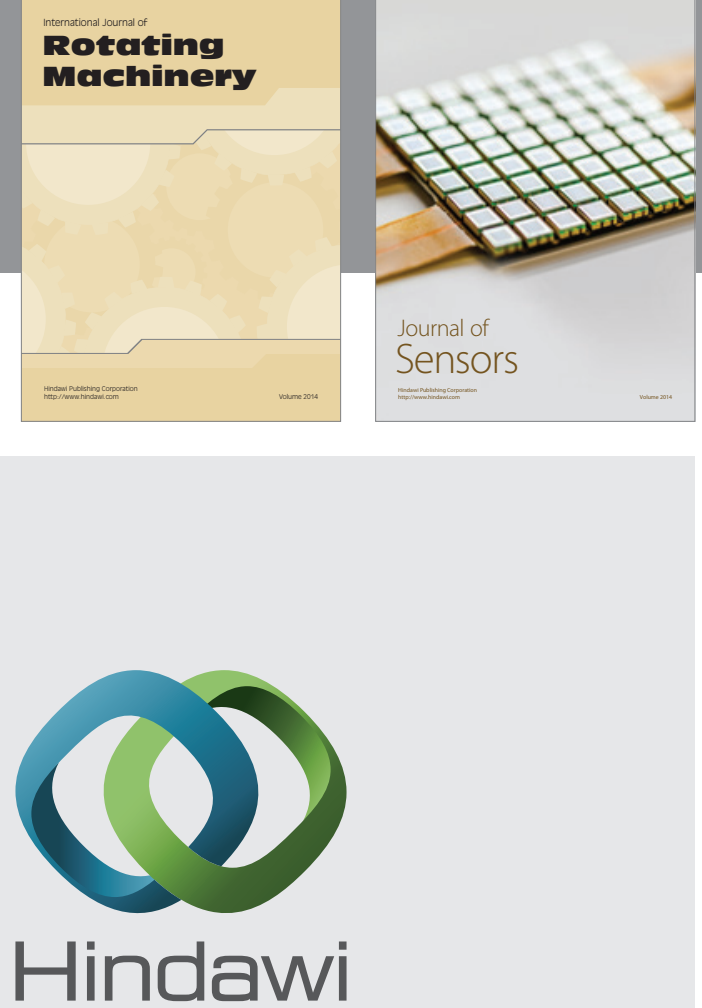

Submit your manuscripts at http://www.hindawi.com
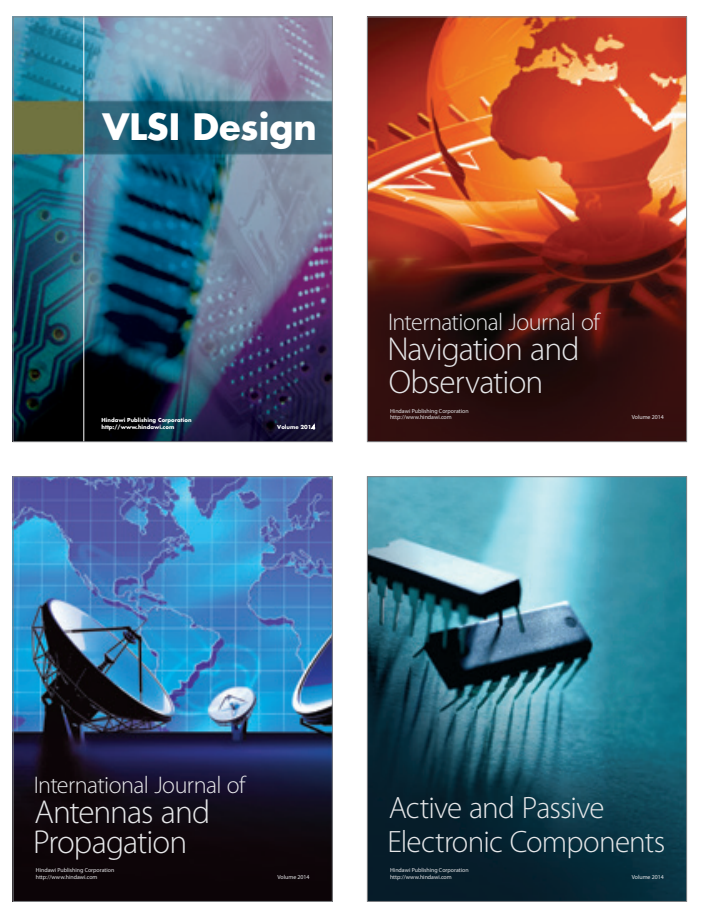
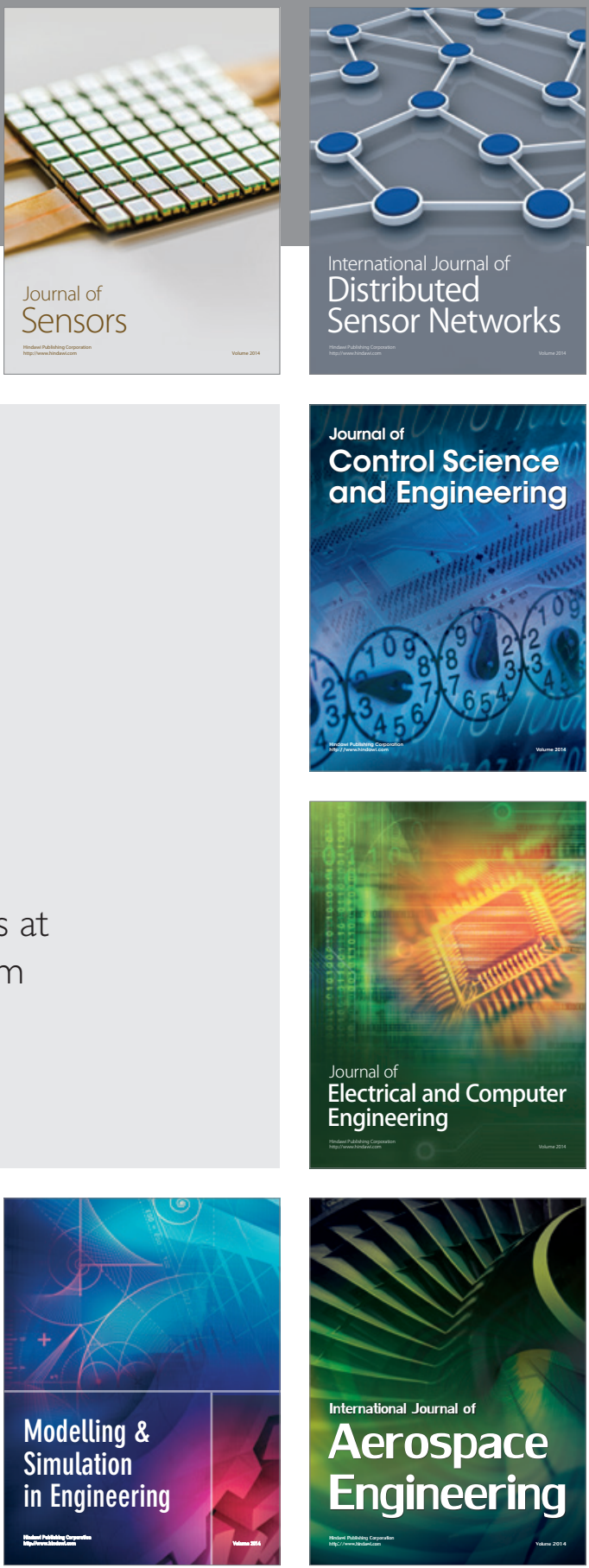

Journal of

Control Science

and Engineering
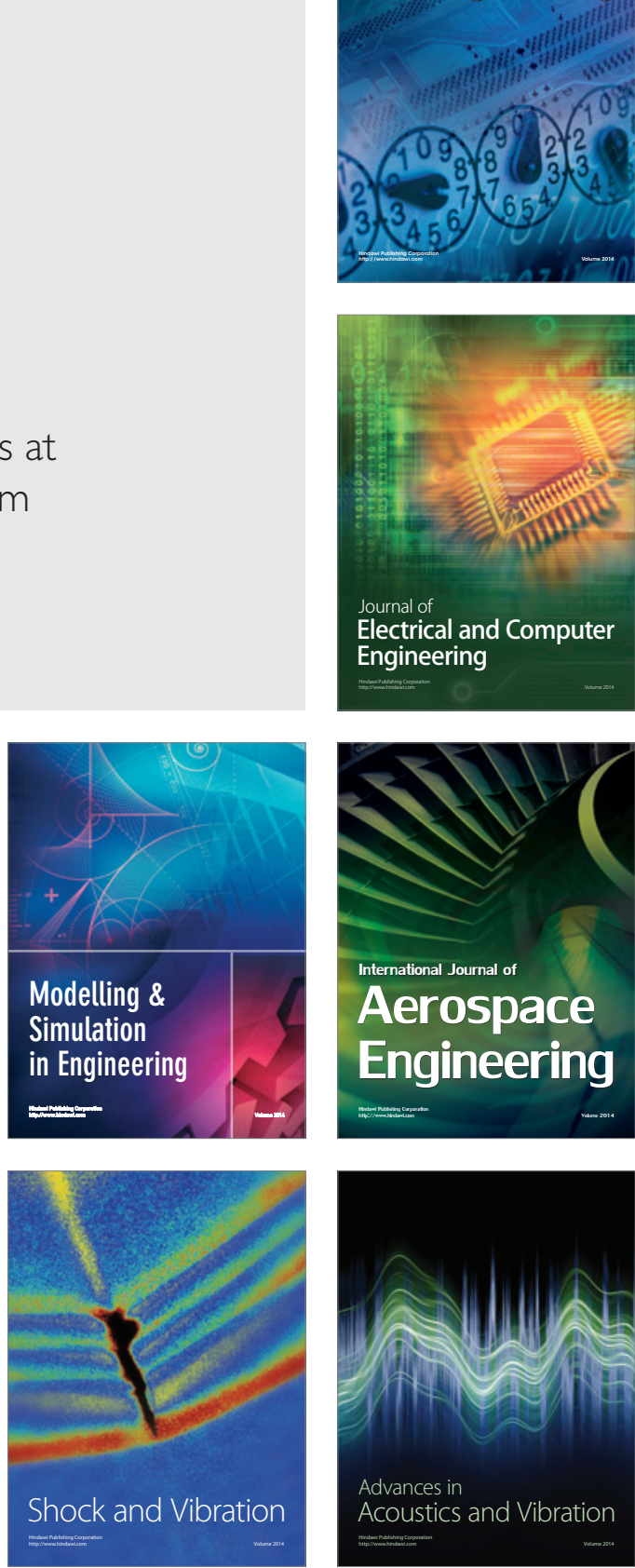